\title{
A Constrained Nonlinear Programming Technique for Interpretation of Self-Potential Anomalies due to Two- Dimensional Inclined Sheets of Finite Depth Extent
}

\author{
J. Asfahani and M. Tlas \\ Atomic Energy Commission, P.O. Box 6901, Damascus, Syria \\ Received: 3/6/2003 Revised: 21/9/2004 Accepted: 18/12/2004
}

\begin{abstract}
A new and simple method based on constrained nonlinear programming concepts is being proposed in this research to interpret the self-potential $(S P)$ anomaly due to two-dimensional inclined sheets of finite depth extent. A constrained nonlinear model has been formulated to describe the geophysical problem related to this buried inclined structure. Thereafter this model has been solved by the famous Frank and Wolfe algorithm in order to estimate the geophysical parameters of the modeled sheets which are: the depth of upper and lower edges $h$ and $H$, inclination angle $\theta$, and electrical dipole moment $k$. This algorithm is chosen for being robust and also because its application to $S P$ data converges rapidly towards the optimal estimation of parameters. To prove the efficiency of this new constrained nonlinear programming technique, three theoretical models with a random noise of $2 \%$ have been studied, and as a result, a very close and satisfactory agreement has been obtained between the assumed and evaluated parameters. The validity of this new proposed method is tested on a practical field example from India, where available $S P$ data existed and has been previously analyzed by a spectral analysis approach. Moreover, a statistical analysis has been carried out to confirm the superiority of this method in comparison with that suggested by Atchuta et al. (1982). All acquired results indicate clearly its precision and accuracy. The advantages of the proposed technique have also been discussed and demonstrated comparing them with other published interpretative techniques.
\end{abstract}

Keywords: Self-potential anomalies, Polarized structures, SP interpretation, Mathematical programming. 


\section{Introduction}

Self-Potential $(S P)$ prospecting is one of the oldest geoelectrical methods and it is still used in many fields of applied geophysics. Several techniques have been proposed and discussed in the literature for interpreting the self-potential anomalies. These techniques are:

1 - Using only a few characteristics points on the anomaly curve. The methods falling in this category were originally developed by Yungul (1950), Paul (1965) and Bhattacharya and Roy (1981). The essential disadvantage of these methods is related to the fact that only a few points are used on the anomaly curve and hence the interpreted results are less reliable, taking into account that in most cases the data is contaminated by noise. However, these methods are considered to be fast and suitable for giving a rough estimation.

2 - Using curve matching techniques Meiser (1962), in which the $S P$ field curve is compared to an album of pre-computed theoretical curves. This process is cumbersoming and the complexity of the method is very high when the variables are numerous and when the process deals with a large number of anomalies.

3 - Using least-squares methods by means of which the final estimation can be found by an iterative procedure (Abdelrahman et al. 1997; Abdelrahman and Sharafldin, 1997).

4 - Using a spectral analysis approach, in which the $S P$ data is analyzed and interpreted in the wave number domain by using the Fourier transform (amplitude and phase spectra), (Atchuta et al. 1982).

More recently, Asfahani and Tlas (2002) developed a constrained nonlinear programming approach to determine the shape of the buried structure from residual $S P$ data.

The main goal of the present work is to implement a new and simple method based on point estimation of geophysical parameters from measured $S P$ data due to two-dimensional inclined sheets of finite depth extent. In fact, estimating the depth to the upper and lower edges $h$ and $H$ of sheets, inclination angle $\theta$, and electrical dipole moment $k$, is a tedious task confronting the mineral industry searching for both sulfides and graphite. The new proposed method is based on the formulation of a constrained nonlinear programming problem solved by using Frank and Wolfe's algorithm (Dantzing 1964; Minoux 1983). This suggested technique has first been tested on theoretical models with random noise and then on a practical field data example taken from India (Atchuta et al. 1982). The accuracy of the result obtained by this constrained nonlinear programming approach depends upon the accuracy to which the residual anomalies can be separated from the observed self-potential data and on the suitability of the model to represent the polarized object. 


\section{Frank and Wolfe Algorithm for the Inversion of SP Data}

Constrained nonlinear programming technique is very famous for its application in many fields of science such as chemical engineering (Bailey et al. 1993; Dantzing 1964), control theory (Biegler 1998), decision theory (Steuer 1986) ... etc. In the present work this technique is applied to the inversion and interpretation of geophysical $S P$ data.

Frank and Wolfe algorithm is considered one of the tools of constrained nonlinear programming technique, and commonly used for solving constrained nonlinear programming problems (Steuer 1986; Frank and Wolfe 1956 \& Minoux 1983). This algorithm is used to solve the constrained nonlinear model that has been formulated to interpret the $S P$ data due to a buried inclined sheet.

Let $f: \mathbf{R}^{\boldsymbol{m}} \rightarrow \mathbf{R}$ be a continuously differentiable function to be minimized over the subset $A \subset \mathbf{R}^{\boldsymbol{m}}$, which is convex and compact in the real space $\mathbf{R}^{\boldsymbol{m}}$. In mathematical form, the problem can be described as follows:

$$
\begin{aligned}
\text { Min } & f(u) \\
\text { subject to } & u \in A
\end{aligned}
$$

Here, $m$ is the number of decision variables and $u$ is the vector of decision variables. To solve this problem, the Frank-Wolfe algorithm proceeds as follows:

1. Start with an initial point $u^{0} \in$ int $(A)$ where int $(A)$ denotes the exact interior of $A$

2. For $k=0,1,2 \ldots$ iterate still the stopping criteria will be verified

i) Determine the feasible (displacement) direction by solving the following linear problem:

$$
\begin{aligned}
& \operatorname{Min}\left[\nabla f\left(u^{k}\right)\right]^{T} u \\
& \text { subject to } u \in A
\end{aligned}
$$

Where $\nabla f$ denotes the gradient vector of the function $f$ and $T$ denotes the vector transpose.

Let $v^{k}$ be an optimal solution. The feasible direction is defined as follows:

$$
d^{k}=v^{k}-u^{k}
$$

ii) Perform a line search in the feasible direction $d^{k}$ by solving the following one-dimensional problem:

$$
\begin{gathered}
\text { Min } f\left(u^{k}+\lambda d^{k}\right) \\
\lambda \in[0,1]
\end{gathered}
$$


Let $\lambda_{k}$ be an optimal solution. Set $u^{k+1}=u^{k}+\lambda_{k} d^{k}$.

iii) Evaluate the stopping criteria (see below); terminate the algorithm if verified; else set $k=k+1$ and return to step i).

\section{Appropriate Stopping Criteria of the Algorithm}

If one of the following criteria is satisfied, then the algorithm will be stopped.

a) $\left|f\left(u^{k+1}\right)-f\left(u^{k}\right)\right|<\varepsilon_{0}$, where $\varepsilon_{0}$ is a small positive predetermined real number.

b) $\|\left(u^{k+1}-u^{k} \|<\varepsilon_{0}\right.$, where $\varepsilon_{0}$ is a small positive predetermined real number and \|\| denotes the $L_{2}$-norm in the space $\mathbf{R}^{\boldsymbol{m}}$.

c) $\left\|\nabla f\left(u^{k}\right)\right\|<\varepsilon_{0}$, where $\varepsilon_{0}$ is a small positive predetermined real number.

\section{Comments on the Algorithm}

1. The Frank-Wolfe algorithm has been chosen for its properties of being robust and converging relatively well towards the optimal solution.

2. If the function $f(u)$ is convex on the subset $A \subset \mathbf{R}^{m}$, then $u^{k}$ is a global minimum.

3. When the assumption of convexity of the function $f(u)$ is not satisfied, this algorithm should be repeated a number of times by starting from a variety of initial trial solutions. The best of the local minima thereby obtained for the problem should be used as the best available approximation of global minima.

\section{Formulating the Geophysical Problem}

The geometry of the inclined sheet of finite depth extent is shown in Fig. 1. The upper and lower edges of the sheet are situated at depth $h$ and $H$ units respectively below the ground surface. The expression for the $S P$ anomaly due to the sheet along a profile perpendicular to its strike is given by Roy and Chowdhury (1959) as follows:

$$
V(x)=\frac{\rho I}{2 \pi} \ln \frac{r_{1}^{2}}{r_{2}^{2}}
$$

Where $I$ is the current per units length.

$\rho$ is the resistivity of the surrounding medium.

$r_{1}, r_{2}$ are the distances of the edges of the sheet from the point of observation.

From Fig. 1, it is clear that: 


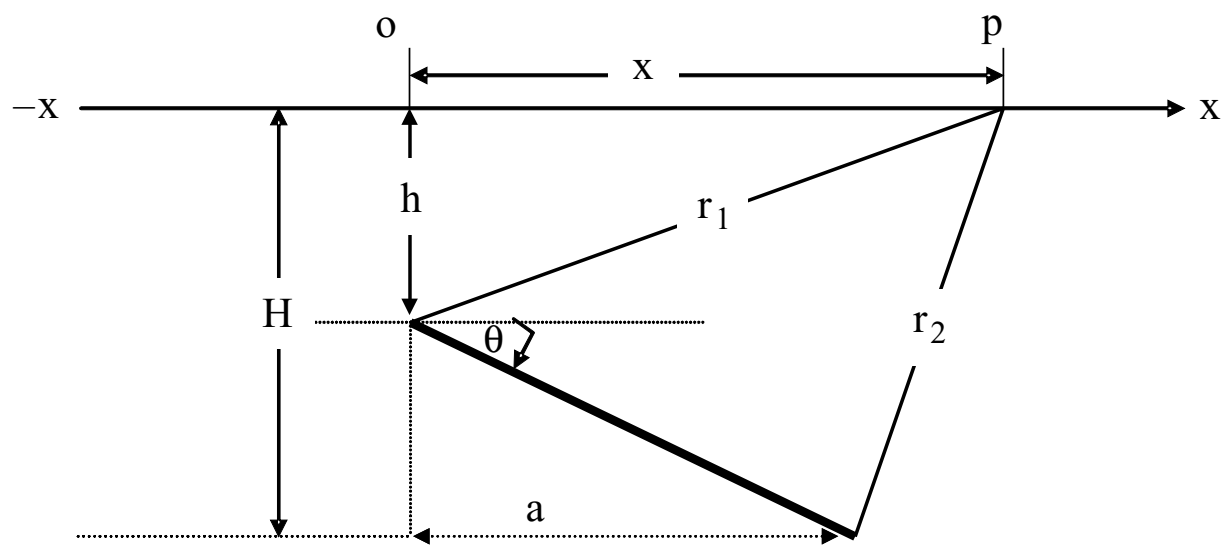

FIG. 1. Cross section of an inclined sheet of finite depth extent (Atchuta et al., 1982).

$$
\begin{aligned}
& r_{1}^{2}=h^{2}+x^{2} \\
& r_{2}^{2}=(x-a)^{2}+H^{2}
\end{aligned}
$$

Where $a=\frac{H-h}{\tan \theta}$

$\theta$ is the inclination angle.

$x$ is the distance of the point of observation from the origin.

Substituting for $r_{1}$ and $r_{2}$ in equation (5), the following equation is obtained:

$$
V(x)=\frac{\rho I}{2 \pi} \ln \frac{x^{2}+h^{2}}{(x-a)^{2}+H^{2}}
$$

Putting $V\left(x_{i}\right)=0$ in equation (6), the following equality is obtained:

$$
x_{0}=\frac{(H+h) \tan ^{2} \theta+(H-h)}{2 \tan \theta}
$$

Putting $x_{i}=0$ in equation (6), the following equality is found:

$$
V(0)=\frac{\rho I}{2 \pi} \ln \frac{h^{2} \tan ^{2} \theta}{H^{2} \tan ^{2} \theta+(H-h)^{2}}
$$

The depth of center $z$ is given by: $\quad z=\frac{H+h}{2}$

The length of the sheet $l$ is given by: $l=\left|\frac{H-h}{\sin \theta}\right|$

The parameters $h, H, \theta$ and $k=\frac{\rho I}{2 \pi}$ are determined at the same time by the inversion technique developed in this research. 
The $S P$ anomaly due to a sheet with $h=2$ units, $H=5$ units, $\theta=60$ degrees, and $k=\rho I / 2 \pi=100 \mathrm{mV}$ was computed using equation (6) and is shown in Fig. 2. The zero anomaly distance $x_{0}$ and the anomaly value at the origin $V(0)$ are also shown in Fig. 2. The evaluation of the inclined sheet parameters $(h, H, \theta, k)$ is the goal of this research work. These parameters can be evaluated by applying the Frank and Wolfe algorithm to the following constrained nonlinear programming problem:

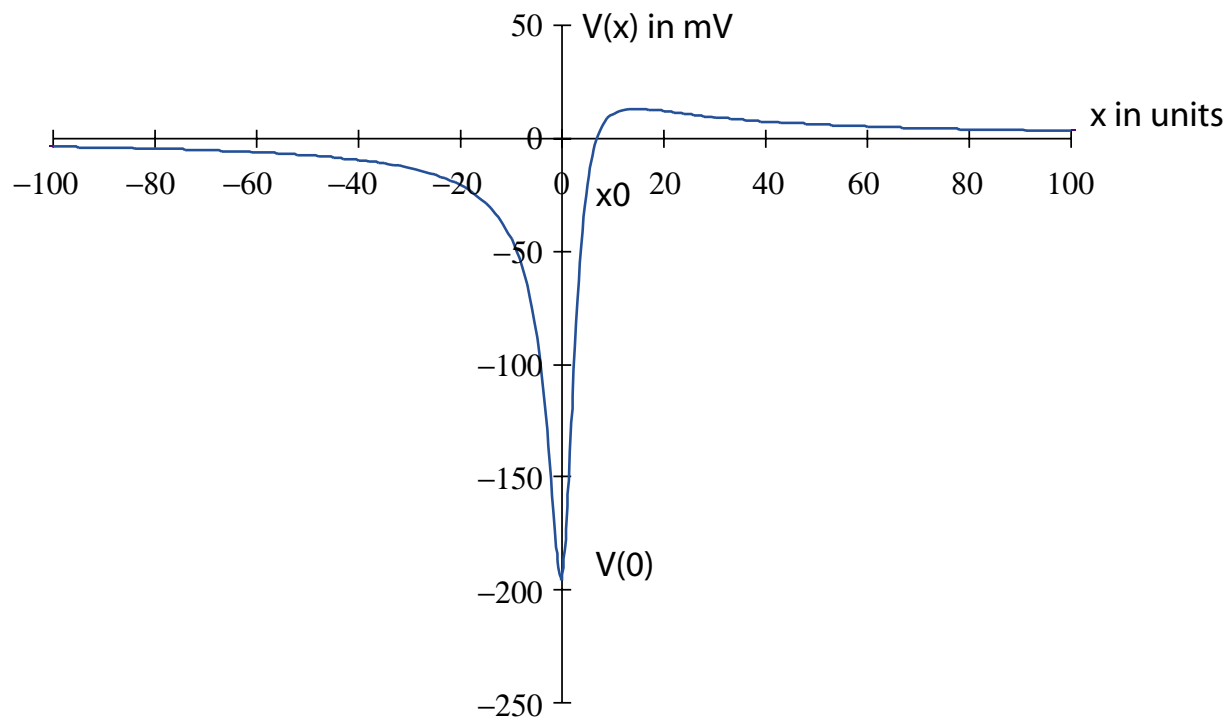

Fig. 2. The $S P$ anomaly $V(x)$ over an inclined sheet of finite depth extent with $h=2$ units, $H=5$ units, $\theta=60$ degrees and $k=100 \mathrm{mV}$.

$$
\min f(h, H, \theta, k)=\min \sum_{i=1}^{N}\left[L\left(x_{i}\right)-V\left(x_{i}, h, H, \theta, K\right)\right]^{2}
$$

Subject to $h \leq h_{\max }$

$$
\begin{aligned}
H & \leq H_{\max } \\
\theta & \leq \theta_{\max } \\
k & \leq k_{\max }
\end{aligned}
$$

$h, H, \theta, k \geq 0$

Where $k=\frac{\rho I}{2 \pi}$ and $L\left(x_{i}\right)$ are the values of the $S P$ anomaly at the points $x_{i}(i$ $=1, \ldots, N)$.

The feasible subset $A$ is defined as follows: 


$$
A=\left\{(h, H, \theta, k) \in R^{4} \backslash h \leq h_{\max }, H \leq H_{\max }, \theta \leq \theta_{\max }, k \leq k_{\max }, h \leq H,(h, H, \theta, k) \geq 0\right\}
$$

This subset is convex and compact. All the necessary conditions for using Frank and Wolfe's algorithm are verified and the solution found by this approach is an optimal solution of problem (9). Introducing the equality (6) into the mathematical program (9), the following constrained nonlinear geophysical programming problem is obtained:

$$
\min f(h, H, \theta, k)=\min \sum_{i=1}^{N}\left[l\left(x_{i}\right)-k \ln \frac{x^{2}+h^{2}}{\left[x-\left(\frac{H-h}{\tan \theta}\right)\right]^{2}+H^{2}}\right]^{2}
$$

Subject to $h \leq h_{\max }$

$$
\begin{aligned}
H & \leq H_{\max } \\
\theta & \leq \theta_{\max } \\
k & \leq k_{\max } \\
h, H, \theta, k & \geq 0
\end{aligned}
$$

The proposed algorithm to be used in the inversion of $S P$ data is adopted where:

$h_{\max }, H_{\max }, \theta_{\max }$ and $k_{\max }$ are 500 meters, 1000 meters, 180 degrees and $10^{6}$ $m V$ respectively. The choice of these values is completely arbitrary and the convergence of our proposed method is not influenced by this choice.

\section{Numerical Examples}

The main objective of the constrained nonlinear programming inversion technique is to get the values of $h, H, \theta$ and $k=\frac{\rho I}{2 \pi}$ related to two- dimensional inclined sheets of finite depth extent. This scheme was applied to both synthetic and real field data from various publications of which one field example is shown.

\section{I - Synthetic Example}

Three data sets were computed for theoretical models with a random error of $2 \%$ analyzed and tested as shown in Table 1 . These three cases were studied for a profile length of 256 meters and a sampling interval of 1-meter. The first model parameters chosen for this study are the following: $h=2$ units, $H=5$ units, $\theta=60$ degree and $k=100 \mathrm{mV}$. The second model parameters are the following: $h=4$ units, $H=10$ units, $\theta=30$ degree and $k=200 \mathrm{mV}$. The third model parameters are the following: $h=6$ units, $H=12$ units, $\theta=75$ degree and 
$k=150 \mathrm{mV}$. The comparison between the assumed and evaluated parameters shows excellent agreement between them. This agreement proves the high efficiency of the proposed constrained nonlinear programming technique.

$\mathrm{T}_{\mathrm{ABLE}} 1$. Results for synthetic data for a profile of $256 \mathrm{~m}$, sampling interval $1 \mathrm{~m}$, with random error of $2 \%$.

\begin{tabular}{|c|c|c|c|c|c|c|c|c|c|c|c|}
\hline \multicolumn{4}{|c|}{ Assumed parameters } & \multicolumn{4}{c|}{ Evaluated parameters } & \multicolumn{3}{|c|}{ Percentage of error } \\
\hline $\begin{array}{c}h \\
\text { units }\end{array}$ & $\begin{array}{c}H \\
\text { units }\end{array}$ & $\begin{array}{c}\theta \\
\text { degrees }\end{array}$ & $\begin{array}{c}k \\
\text { mili volt }\end{array}$ & $\begin{array}{c}h \\
\text { units }\end{array}$ & $\begin{array}{c}H \\
\text { units }\end{array}$ & $\begin{array}{c}\theta \\
\text { degrees }\end{array}$ & $\begin{array}{c}k \\
\text { mili volt }\end{array}$ & in $h$ & in $H$ & in $\theta$ & in $k$ \\
\hline 2 & 5 & 60 & 100 & 2.001 & 4.998 & 60.004 & 102.075 & 0.050 & 0.040 & 0.006 & 2.075 \\
4 & 10 & 30 & 200 & 3.999 & 9.999 & 29.998 & 203.982 & 0.025 & 0.010 & 0.006 & 1.991 \\
6 & 12 & 75 & 150 & 6.002 & 11.99 & 74.990 & 153.208 & 0.033 & 0.058 & 0.013 & 2.138 \\
\hline
\end{tabular}

\section{II - Field Example}

The proposed technique for the inversion of $S P$ data has been applied to interpret the $S P$ anomaly (Fig. 3) obtained across a mineralized belt in the Kalava fault zone, $52 \mathrm{~km}$ south of Kurnool in the Cuddapah Basin, Andhra, Pradesh, India (Sanker Narayan et al., 1982). Earlier drilling of some anomalies in this area by the Geological Survey of India encountered carbonaceous shales with sulfide mineralization. These might be the sources causing the $S P$ anomaly. The $S P$ profile having the length of 255 meters has been digitized at an interval of 6.375 meters (1unit $=6.375$ meters) and subjected to interpretation by the new approach. The evaluated parameters obtained by the constrained nonlinear programming technique are shown in Table 2. The depth to the top and bottom $(h, H)$ are 3.064 units and 8.029 units respectively. The inclination angle $\theta$ and the electrical dipole moment $k$ are evaluated as equal to 105 degrees and $57 \mathrm{mV}$ respectively. Using these evaluated parameters, the theoretical profile has been computed for direct comparison with the field data in Fig. 3. This comparison indicates clearly the close match between the observed and the computed anomalies. The depth to the target $(h)$ obtained by Sanker Narayan et al. (1982) method is 17 meters. Table 2 and Fig. 3 show comparison of the results obtained by our constrained nonlinear programming approach and those obtained by Atchuta et al. (1982) who used a Fourier transform method. Both results will be statistically analyzed and discussed to demonstrate the advantages and the superiority of our proposed technique.

\section{Statistical Analysis and Comparison Study}

To confirm the efficiency of our proposed method in comparison with the method of Atchuta et al. (1982), the standard errors and the confidence intervals for both methods have been computed. Following that, the statistical Fisher test has been applied. 


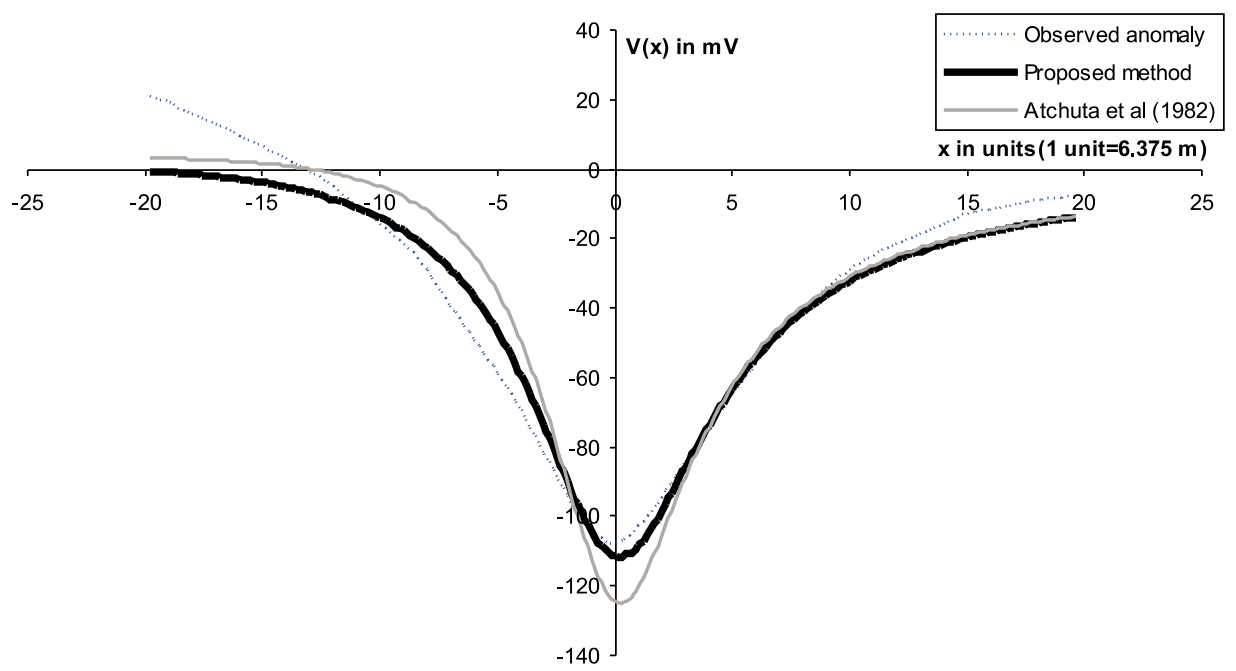

FIG. 3. SP anomaly over a sulfide body in the Kalava fault zone (Cuddapah basin, India). The theoretical computed anomalies are also shown for both methods.

TABLE 2. Comparison of results using two different interpretation methods.

\begin{tabular}{|l|c|c|}
\hline \multicolumn{1}{|c|}{ Evaluated parameters } & Interpretation of Atchuta et al. (1982) & Present method \\
\hline$h$ in meters & 15.9 & 19.345 \\
\hline$H$ in meters & 41.2 & 51.186 \\
\hline$\theta$ in degrees & 110 & 105 \\
\hline$k$ in $m V$ & 63.68 & 57 \\
\hline$x_{0}$ in meters & - & -135.417 \\
\hline$V(0)$ in $m V$ & - & -111.386 \\
\hline$z$ depth of center in meters & - & 35.360 \\
\hline$l$ length of sheet in meters & - & 32.782 \\
\hline
\end{tabular}

The standard error (i.e., standard deviation of residuals) is given by the following equality:

$$
S=\sqrt{\frac{\sum_{i=1}^{N}\left[L\left(x_{i}\right)-V\left(x_{i}, h, H, \theta, k\right]^{2}\right.}{N-P}}
$$

Where $P$ is the number of independent parameters. In our method $P=4$ (i.e., $H, H, \theta, k)$ and $N$ is equal to 198 discrete points. Using this equation, the standard error for our method is found to be equal to $S_{1}=8.053$ and the standard error for Atchuta et al. (1982) is found to equal to $S_{2}=10.862$. 
For a confidence level of $1-\alpha=\% 95(\alpha=0.05)$, the confidence interval is given by the following inequalities:

$$
V\left(x_{i}, h, H, \theta, k\right)-Z_{\frac{\alpha}{2}} \times S \leq L\left(x_{i}\right) \leq V\left(x_{i}, h, H, \theta, k\right)+Z_{\frac{\alpha}{2}} \times S
$$

Where $Z_{\frac{\alpha}{2}}=Z_{0.025}=1.96$ (Z-test is generally used for a large number of measurements $N \geq 30$ ).

For our method, this interval is found to be:

$$
\begin{aligned}
& V\left(x_{i}, h, H, \theta, k\right)-1.96 \times 8.053 \leq L\left(x_{i}\right) \leq V\left(x_{i}, h, H, \theta, k\right)+1.96 \times 8.053 \Rightarrow \\
& V\left(x_{i}, h, H, \theta, k\right)-15.784 \leq L\left(x_{i}\right) \leq V\left(x_{i}, h, H, \theta, k\right)+15.784 \quad(i=1, \ldots, 198)
\end{aligned}
$$

From the above inequalities, it is clear that $95 \%$ of the $S P$ field measurements belong to this interval.

Applying the same steps for Atchuta et al. (1982) results, the confidence interval is found to be equal to:

$$
V\left(x_{i}, h, H, \theta, k\right)-21.290 \leq L\left(x_{i}\right) \leq V\left(x_{i}, h, H, \theta, k\right)+21.290 \quad(i=1, \ldots, 19
$$

It is clear from this comparison between these two methods that the standard error calculated for our method is less than that the standard error for the method of Atchuta et al. In addition, the confidence interval related to our method is completely included in the confidence interval of Atchuta et al. which indicates the improved accuracy of our proposed interpretative method.

To establish the preciseness of our method, a Fisher test has been implemented as follows:

Suppose $\sigma_{1}$ and $\sigma_{2}$ are the population standard errors (the population here is the field measurements when the number of measurements $N$ tends to the infinity $(N \rightarrow \infty)$ ) for both our method and Atchuta et al., method respectively.

Suppose also the null hypothesis $H_{0}: \sigma_{1}^{2}=\sigma_{2}^{2}$ (i.e., the two methods have the same precision) is examined against the alternative hypothesis $H_{1}: \sigma_{1}^{2}=\sigma_{2}^{2}$ (i.e., our method is more precise than that of Atchuta et al.).

The Fisher statistic is given by $F=\frac{S_{1}^{2}}{S_{2}^{2}}=\frac{8.053^{2}}{10.862^{2}}=0.55$.

The critical value for $95 \%$ confidence level is $f_{1-a}\left(v_{1}, v_{2}\right)=f_{0.95}(197,197)=$ 1.26 , 
where $v_{1}, v_{2}$ are the freedom degrees $\left(v_{1}=v_{2}=N-1\right)$.

The rejection region is: $F \leq f_{1-a}\left(v_{1}=v_{2}\right)=f_{0.95}(197,197)$ and the acceptance region is:

$$
F>f_{1-a}\left(v_{1}, v_{2}\right)=f_{0.95}(197,197)
$$

Having that, the value of 0.55 belongs to the rejection region $(0.55<1.26)$. The null hypothesis $H_{0}$ is therefore rejected, and consequently the alternative hypothesis $H_{1}$ is accepted. As a conclusion it follows that $\sigma_{1}<\sigma_{2}$ which means that, our method is more precise than that of Atchuta et al. (1982).

\section{Discussion and Conclusion}

In this paper, a new geophysical method is being proposed for the interpretation of $S P$ anomalies related to buried inclined sheets of finite depth extent. The proposed method is based on the constrained nonlinear programming concept. First of all, a constrained nonlinear model has been formulated to describe the geophysical problem related to the buried inclined sheet. This constrained nonlinear geophysical model has been solved by using the robust Frank and Wolfe algorithm. Its application to the $S P$ data converges rapidly towards an optimal estimation of parameters. The utilization of the new proposed constrained nonlinear programming technique easily leads to the estimation of the inclined sheet parameters, i.e., depth to upper and lower edges $h$, and $H$, inclination angle $\theta$, and electrical dipole moment $k$. The advantages of the constrained nonlinear programming technique over other interpretative techniques which only use a few points, distances, and nomograms, are: 1) All observed values are used during the interpretation process. 2) The technique is automatic. 3) The four parameters $(h, H, \theta, k)$ are determined at the same time by finding an optimal solution. The proposed approach has been validated with synthetic and real data. The application of the constrained nonlinear programming technique to a set of practical field data resulted in good agreement between observed and computed data. Statistical analysis indicates also the precision of our method and its superiority, in comparison with other interpretative methods. Therefore, the proposed technique can be used for routine analysis of $S P$ data to determine the parameters with ease and accuracy.

\section{Acknowledgment}

The authors would like to thank Prof. I. Othman Director General of the Atomic Energy Commission of Syria for his interest and continuous encouragement to achieve this work. Special thanks to anonym reviewers for their constructive remarks and appreciated suggestions which considerably improve the quality of this paper. 


\section{References}

Abdelrahman, E.S.M. and Sharafeldin, S.M. (1971) A least-squares approach to depth determination from self-potential anomalies caused by horizontal cylinders and spheres, Geophysics, 62: 44-48.

Abdelrahman, E.M., El-Araby, T.M., Ammar, A.A. and Hassanein, H.I. (1997) A leastsquares approach to shape determination from residual self-potential anomalies, Pure Appl. Geophys., 150: 121-128.

Asfahani, J. and Tlas, M. (2002) A nonlinear programming technique for the interpretation of self-potential anomalies, Pageoph, 159: 1333-1343.

Atchuta Rao, Ram Babu and Sivakumar Sinha, G.D.J. (1982) A Fourier transform method for the interpretation of self-potential anomalies due to two to two-dimensional inclined sheets of finite depth extent, Pageoph, 120: 365-374.

Atchuta Rao, D. and Ram Babu, H.V. (1983) Quantitative interpretation of SP anomalies due to two-dimensional sheet-like bodies, Geophysics, 48: 1659-1664.

Bailey, J.K., Hrymak, A.N., Treiber, S.S. and Hawkins, R.B. (1993) Nonlinear optimization of a hydrocracker fractionation plant, Comput. Chem. Engng., 17: 123.

Bhattacharya, B.B. and Roy, N. (1981) A note on the use of nomogram for self-potential anomalies, Geophysical Prospecting, 29: 102-107.

Biegler, L.T. (1998) Advances in nonlinear programming concept for process control, J. Process Control, 8: 301.

Dantzing, G.B. (1964) Linear Programming and Extension, Princeton University Press.

Frank, M. and Wolfe, P. (1956) An algorithm for quadratic programming, Naval Research Logistics Quarterly, 3: 95-110.

Meiser, P. (1962) A method of quantitative interpretation of self-potential measurements, Geophys. Prosp., 10: 203-218.

Minoux, M. (1983) Programation Mathematique (Theorie et Algorithmes), tomeu 1.

Paul, M.K. (1965) Direct interpretation of self-potential anomalies caused by inclined sheets of infinite extension, Geophysics, 30: 418-423.

Roy, A. and Chowdhury, D.K. (1959) Interpretation of self-potential data for tabular bodies, $J$. Sci. Engng. Res., 3: 35-54.

Sanker Narayan, P.V. et al. (1982) Report on multiparameter geophysical experiment in Kalava area (Cuddaph basin) Kurnool district, Andhra Pradesh, The Fifth Workshop on Status, Problem and Programs in Duddapah Basin, 11-12 January, 1982, Organized by the Institute of Peninsular Geology, Hyderabad, India.

Steuer, R. (1988) Multiple Criteria Optimization: Theory, Computation, and Application, John Wiley \& Sons.

Yungul, S. (1950) Interpretation of spontaneous polarization anomalies caused by spherical ore bodies, Geophysics, 15: 237-246. 


\section{تقنية البرمجة غير الخطية المقيدة لتفسير شاذات الكمون الذاتي العائدة لصفائح مائلة ثنائية البعد وذات امتداد محدود}

\section{جمال أصفهاني ، و محمد طلاس}

هيئة الطاقة الذرية ، ص .ب : 19 •7 ، دمشق - سورية .

المستخلص . تم في هذه الورقة اقتراح طريقة جديدة وسهلة تعتمد

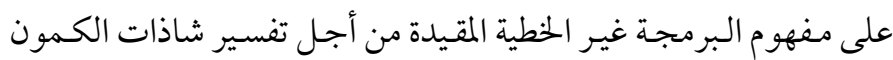

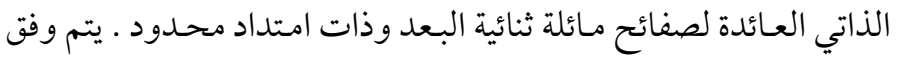

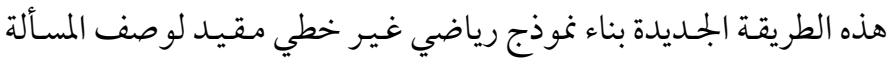

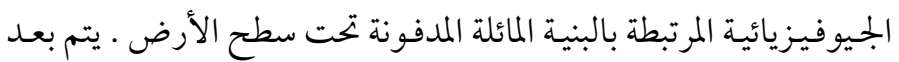

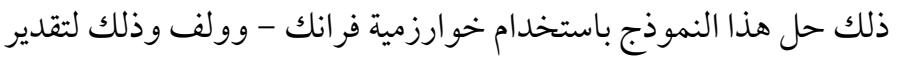

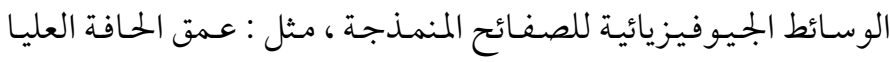

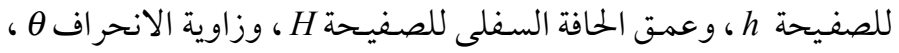

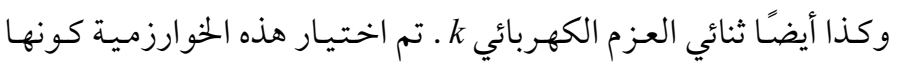

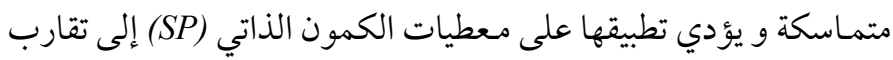

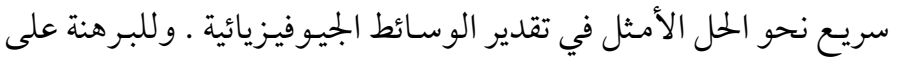

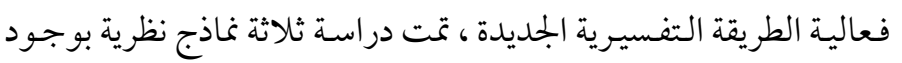

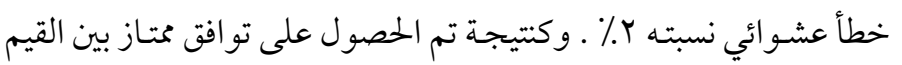

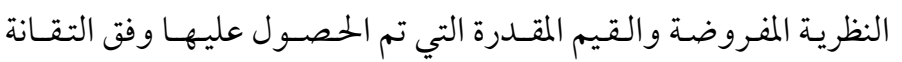

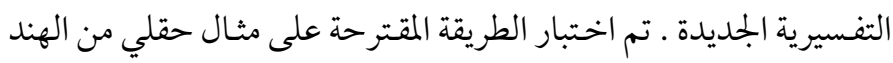

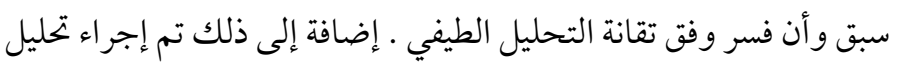

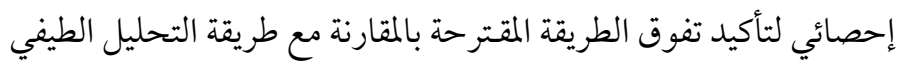

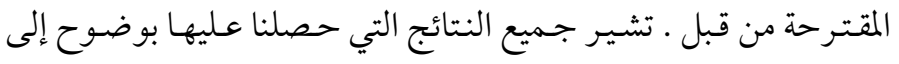

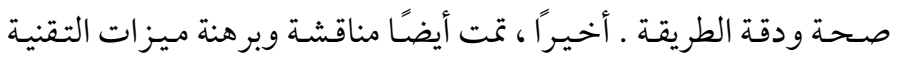

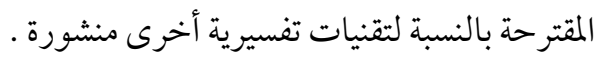

